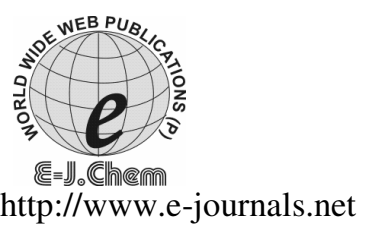

\title{
Synthesis and Antifungal Activity of Azetidinone and Thiazolidinones Derivatives of 2-Amino-6-(2-naphthalenyl)thiazolo[3,2-d]thiadiazole
}

\author{
K.H.PATEL and A.G.MEHTA* \\ Department of Chemistry \\ P.T.Science College \\ Surat, Gujarat, India
}

Received 7 July 2006; Accepted 22 August 2006

\begin{abstract}
: 2-amino-6-(2-naphthalenyl)thiazolo[3,2-d]thiadiazole [1] was prepared by treatment of KCNS and $\mathrm{Br}_{2}$ on 2-Amino-4-(2-naphthalenyl) thiazole. This amine on facile condensation with aromatic aldehydes afford Schiff Base/anils/azomethines(2a-h). These anils on cyclocondensation reaction with chloro acetyl chloride and thio glycolic acid (i.e. mercapto acetic acid) afford 2azetidinones and 4-thiazolidinones respectively. The prepared compounds have been screened on some stains of fungai.
\end{abstract}

Keywords: 2-Azetidinones, 4-thiazolidinones, cyclo-condenction reaction, facile condection, spectral studies antibacterial action.

\section{Introduction}

Thiazoles are one of the most intensively investigated classes of aromatic five membered hetrocycles. Thiazole derivatives find a variety of applications ranging from bacteriostatics, antibiotics, CNS regulants of high selling diureties ${ }^{1-5}$. All these facts were driving force to develop novel thiozole derivatives with wide structural varation ${ }^{6}$. Thus thiazole derivatives playes pivotal role in medicinal chemistry.

As part of interest in hetrocycles that have been explored for developing pharmaceutically important molecules, 4-thiazolidinones ${ }^{7-9}$ and 2 -azetidinones ${ }^{10-13}$ have played an important role in medicinal chemistry. Moreover they have been studied extensively because of their ready accessibility, diverse chemical reactivity and broad spectrum of 
biological activity. The area in which the transformation of 2-amino-4-(2-naphthalenyl) thiazole into azetidinones and thiazolidinones has been reported recently by us ${ }^{14}$. In continuous at this work ${ }^{14}$ the present paper comprises the hetereocyclization of 2-amino-4(2-naphthalenyl) thiazole into fused hetereocyclic amine and its further transformation into 2-azetidinones and 4-thiazolidinones. The work is scanned in Scheme-1.

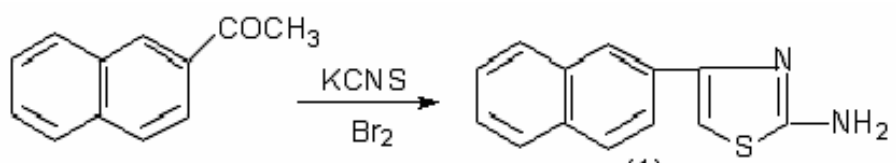

(1)

2-Acetyl naphthalene

2-amino-4-(2-naphthalenyl) thiazole

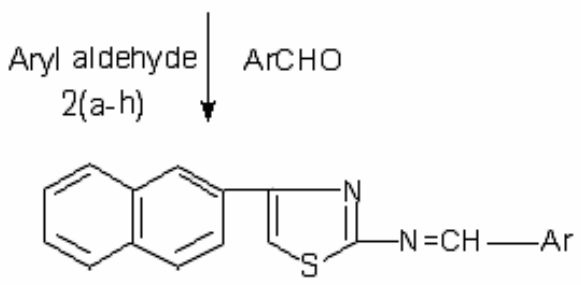

Schiff Base

$(3 a-h)$
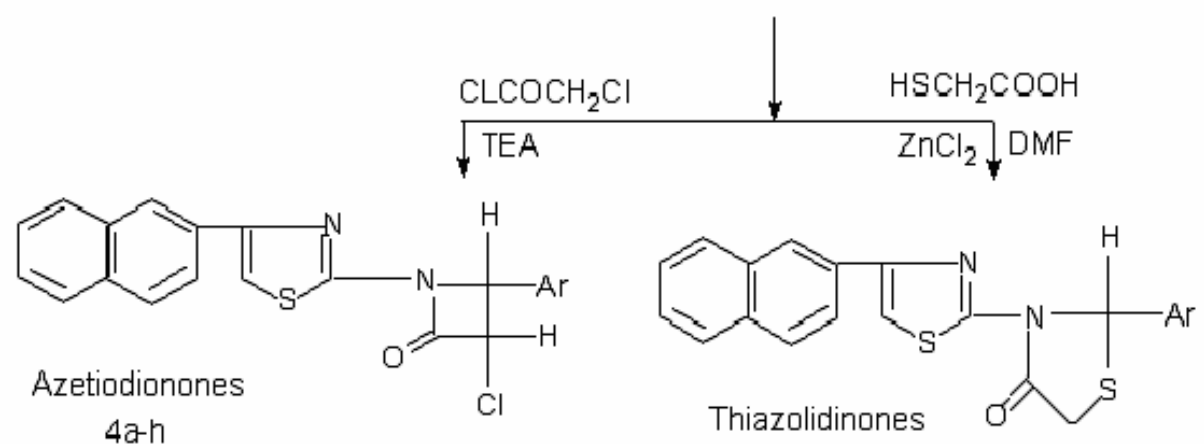

$5 \mathrm{a}-\mathrm{h}$

where Ar:

\section{Scheme-1}

a) Phenyl , b) 4-Methoxy Phenyl c) 4-Hydroxy Phenyl d) 2-Hydroxy Phenyl

e) 4-Methyl Phenyl f) 3,4-Methylenedioxy Phenyl, g) 4-Hydroxy-3-Methoxy Phenyl, h) 3,4-Diethoxy Phenyl

\section{Experimental}

\section{Materials}

2-Amino-4-(2-naphthalenyl) thiazole was prepared according to method reported ${ }^{15}$. The aromatic benzadelydes (2a-h) viz; a: benzaldehyde, b: 4-methyl benzaldehyde, c: 4-hydroxy benzaldehyde, d: 2- hydroxybenzaldehyde, e: 4- methoxy benzaldehyde, f: 4bromobenzaldelhyde, g: 3,4-ethylenedioxy benzaldehyde, h: 4-hydroxy-3-methoxy benzaldehyde and i: 3,4-diethoxy benzaldehyde were obtained from local dealer. All other chemicals used where of laboratory grade. 


\section{Measurements}

Melting points were determined in open capillary tubes and were uncorrected. The IR spectra were recorded in KBR pellets on a nicolet 760D spectrophotometer and 1H NMR spectra in $\mathrm{CDCI}_{3}$ on Perkin Elmar NMR spectrometer using TMS as an internal standard. Antifungal activity of all the compounds were studied against various fungi (Tables-3 \& 4) at a concentration of $100 \mathrm{ppm}$ by agar cup method ${ }^{16}$. Methanol system was used as control in the method. Under similar conditions using penicillin and sulfanilamide as a standard. The comparison carried at control experiment. The area of inhibition of zone is measured as percentage.

\section{Preparation of 2-amino-6-(2-naphthalenyl)thiazolo[3,2-d]thiadiazole}

In a three necked flask, a solution of 2-amino-4-(2-naphthalenyl) thiazole (0.02 mole) in 1,4dioxane was placed. Then the flask was kept in an ice-bath. The mechanical stirrer was fitted. The KCNS (0.08 mole) was added gradually into the solution with constant stirring. Finally $\mathrm{Br}_{2}(16 \mathrm{ml})$ in acetic acid $(100 \mathrm{ml})$ was added slowly with constant stirring. The whole assembly with stirrer was kept in an ice-bath for 6 hrs. The resultant mixture was kept aside until reached room temperature. The product was poured into ice-water, filtered and washed with 1,4-dioxane. The product thus was recrystallized by 1,4-dioxane-ethanol [50:50(v/v)] mixture and checked on TLC. Finally the product was purified by column chromatography over silica gel using ethyl acetate: benzene (30:70) as an eluent. Yield was $48 \%$. M.p. $156-7^{0} \mathrm{C}$ uncorrected.

\begin{tabular}{lllll}
\multicolumn{5}{c}{ Analysis: $\mathrm{C}_{14} \mathrm{H}_{11} \mathrm{~N}_{3} \mathrm{~S}_{2}(285)$} \\
\hline \multicolumn{6}{c}{$\mathrm{C} \%$} & $\mathrm{H} \%$ & $\mathrm{~N} \%$ & $\mathrm{~S} \%$ \\
\hline Cal. & 58.94 & 3.86 & 14.73 & 22.45 \\
Fin. & 58.8 & 3.75 & 14.6 & 22.3 \\
\hline
\end{tabular}

IR : $\quad 3400-3200 \mathrm{~cm}^{-1} \mathrm{NH}_{2}, 3030,1600,1100 \mathrm{~cm}^{-1}$ aromatic, $1550 \mathrm{~cm}^{-1} \mathrm{C}=\mathrm{N}$

NMR : $\quad \delta=7.2-8.9$ ppm multiplate aromatic $8 \mathrm{H}, \delta=4.1 \mathrm{ppm}, \mathrm{S} \mathrm{NH}_{2}$

\section{Preparation of Schiff base (3a-h)}

\section{General procedure}

A mixture of equimolar amount (0.01) of 2-amino-6-naphthalenylthiazolo [3,2-d] thiadiazole (1), benzaldehyde derivative (2a-h) in ethanol : 1,4-dioxane (50:50) (40 ml) and piperidine $(0.3 \mathrm{ml})$ was refluxed for $5 \mathrm{hrs}$ on water bath. The reaction mixture was concentrated, cooled and poured in water; the solid obtained was filtered and recrystallised from ethanol to give Schiff base (3a-h). It was obtained in $60-65 \%$ yield.

\section{Preparation of 2-Azetidionones (4a-h)}

\section{General Procedure}

A mixture of Schiff base (3a-h) $(0.002 \mathrm{~mol})$ and triethyl amine [TEA] $(0.004 \mathrm{~mol})$ was dissolved in 1,4-dioxane $(50 \mathrm{ml})$, cooled and stirred. To this well-stirred cooled solution chloro acetyl chloride $(0.004 \mathrm{mmol})$ was added drop wise with in a period of $20 \mathrm{~min}$. The reaction mixture was then stirred for an additional $3 \mathrm{hrs}$ and left at room temperature for 48 hrs. The resultant mixture was concentrated, cooled, poured in to ice cold water, filter and then dired. The product thus obtained was purified by column chromatography over silica gel using 30\% ethyl acetate: $70 \%$ benzene as an eluent. Recrystallization from either / $\mathrm{n}$-Hexane gave 2 -azetidinones (4a-h), which were obtained in $45-55 \%$ yield. 
Table 1. Analytical and spectral Data of Commands 4a-h

\begin{tabular}{|c|c|c|c|c|c|c|c|c|c|c|c|c|c|}
\hline \multirow{3}{*}{ 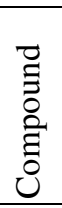 } & \multirow{3}{*}{$\begin{array}{l}\text { Molecular } \\
\text { Formula }\end{array}$} & \multirow{3}{*}{$\begin{array}{c}\text { Mol. } \\
\text { Wt }\end{array}$} & \multirow{3}{*}{$\frac{20}{00}$} & \multirow{3}{*}{$\begin{array}{c}\text { M.P.* } \\
\left({ }^{\circ} \mathrm{C}\right)\end{array}$} & \multicolumn{8}{|c|}{$\%$ Analysis } & \multirow{3}{*}{$\operatorname{PMR}(\delta \text { PPM })^{* *}$} \\
\hline & & & & & \multicolumn{2}{|r|}{$\mathrm{C}$} & \multicolumn{2}{|c|}{$\mathrm{H}$} & \multicolumn{2}{|r|}{$\mathrm{N}$} & \multicolumn{2}{|c|}{ S } & \\
\hline & & & & & Cald & Found & Cald & Found & Cald & Found & Cald & Found & \\
\hline $4 a$ & $\mathrm{C}_{23} \mathrm{H}_{16} \mathrm{ClN}_{3} \mathrm{OS}_{2}$ & 449.5 & 555 & 5193.4 & 61.4 & 61.3 & 3.56 & 3.5 & 9.34 & 9.2 & 14.23 & 14.1 & $\begin{array}{l}\text { 9-2 1 } \mathrm{H} \mathrm{d} \mathrm{C}_{3} \mathrm{H} 9-2-7-8(13 \mathrm{H} \\
\text { mtd aromatic, } \mathrm{C}_{4} \mathrm{H} \text { and } \mathrm{S} \text { of } \\
\text { thiazole- } \mathrm{CH} 9.2\left(\mathrm{H} \mathrm{d} \mathrm{C}_{3} \mathrm{H}\right)\end{array}$ \\
\hline $4 b$ & $\mathrm{C}_{24} \mathrm{H}_{18} \mathrm{ClN}_{3} \mathrm{OS}_{2}$ & 463.5 & 55 & $181-2$ & 62.13 & 62.0 & 3.88 & 3.8 & 9.06 & 9.00 & 13.8 & 13.7 & $\begin{array}{l}9.2\left(\mathrm{H} \mathrm{d} . \mathrm{C}_{3} \mathrm{H}\right) \\
1.9\left(3 \mathrm{H}_{2} \mathrm{CH}\right)\end{array}$ \\
\hline $4 c$ & $\mathrm{C}_{23} \mathrm{H}_{16} \mathrm{ClN}_{3} \mathrm{O}_{2} \mathrm{~S}_{2}$ & 465.5 & 50 & $175-6$ & 59.29 & 59.2 & 3.43 & 3.4 & 9.02 & 8.9 & 13.74 & 13.6 & $\begin{array}{l}9.2\left(\mathrm{H} \mathrm{d} . \mathrm{C}_{3} \mathrm{H}\right) \\
3.9(\mathrm{H} \mathrm{S} \mathrm{OH})\end{array}$ \\
\hline $4 d$ & $\mathrm{C}_{23} \mathrm{H}_{16} \mathrm{ClN}_{3} \mathrm{O}_{2} \mathrm{~S}_{2}$ & 465.5 & 55 & $203-4$ & 59.29 & 59.2 & 3.43 & 3.4 & 9.02 & 8.9 & 13.74 & 13.6 & $\begin{array}{l}9.2\left(\mathrm{H} \mathrm{d} . \mathrm{C}_{3} \mathrm{H}\right) \\
3.9(\mathrm{H} \mathrm{S} \mathrm{OH})\end{array}$ \\
\hline $4 e$ & $\mathrm{C}_{24} \mathrm{H}_{18} \mathrm{ClN}_{3} \mathrm{O}_{2} \mathrm{~S}_{2}$ & 479.5 & 50 & $163-4$ & 60.06 & 59.9 & 3.75 & 3.6 & 8.76 & 8.6 & 13.34 & 13.2 & $\begin{array}{l}9.2\left(\mathrm{H} \mathrm{d} . \mathrm{C}_{3} \mathrm{H}\right) \\
2.1\left(3 \mathrm{H}_{3} \mathrm{OCH}_{3}\right)\end{array}$ \\
\hline $4 \mathrm{f}$ & $\mathrm{C}_{24} \mathrm{H}_{16} \mathrm{ClN}_{3} \mathrm{O}_{3} \mathrm{~S}_{2}$ & 493.5 & 52 & $171-2$ & 58.35 & 58.2 & 3.24 & 3.7 & 8.51 & 8.4 & 12.96 & 12.85 & $\begin{array}{l}9.2\left(\mathrm{H} \mathrm{d} . \mathrm{C}_{3} \mathrm{H}\right) \\
3.9\left(2 \mathrm{H} \mathrm{of} \mathrm{CH}_{3}\right)\end{array}$ \\
\hline $4 \xi$ & $\mathrm{C}_{24} \mathrm{H}_{18} \mathrm{ClN}_{3} \mathrm{O}_{3} \mathrm{~S}_{2}$ & 495.5 & 50 & $200-1$ & 58.12 & 58 & 3.63 & 3.5 & 8.47 & 8.3 & 12.91 & 12.8 & $\begin{array}{l}9.2\left(\mathrm{H} \mathrm{d} . \mathrm{C}_{3} \mathrm{H}\right) \\
2.1\left(3 \mathrm{H}_{3} \mathrm{OCH}\right)\end{array}$ \\
\hline $4 h$ & $\mathrm{C}_{25} \mathrm{H}_{20} \mathrm{ClN}_{3} \mathrm{O}_{3} \mathrm{~S}_{2}$ & 509.5 & 45 & $180-1$ & 58.8 & 58.7 & 3.92 & 3.8 & 8.24 & 8.1 & 12.56 & 12.4 & $\begin{array}{l}9.2\left(\mathrm{H} \mathrm{d} . \mathrm{C}_{3} \mathrm{H}\right) \\
2.5\left(\mathrm{q}, 4 \mathrm{H} \text { of } \mathrm{CH}_{2}\right) \\
2.0\left(\mathrm{t}, 6 \mathrm{H} \text { of } \mathrm{CH}_{3}\right)\end{array}$ \\
\hline
\end{tabular}

\footnotetext{
* Uncorrected ${ }^{* *}$ All the NMR spectra containing multiplate between 7.8 to 8.2 which assigned aromatic ring $+\mathrm{C}_{4} \mathrm{H}$ and thiazole $\mathrm{CH}$.
} 
Table 2. Analytical and spectral Data of Commands Thiazolidinones, 5a-h

\begin{tabular}{|c|c|c|c|c|c|c|c|c|c|c|c|c|c|}
\hline \multirow{3}{*}{ 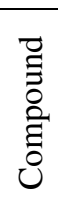 } & \multirow{3}{*}{$\begin{array}{l}\text { Molecular } \\
\text { Formula }\end{array}$} & \multirow{3}{*}{$\begin{array}{l}\text { Mol. } \\
\text { Wt }\end{array}$} & \multirow{3}{*}{ 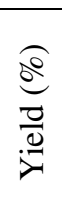 } & \multirow{3}{*}{$\begin{array}{l}\text { M.P. } \\
\left({ }^{\circ} \mathrm{C}\right)^{*}\end{array}$} & \multicolumn{8}{|c|}{$\%$ Analysis } & \multirow{3}{*}{$\operatorname{PMR}(\delta \text { PPM })^{* *}$} \\
\hline & & & & & \multicolumn{2}{|c|}{$\mathrm{C}$} & \multicolumn{2}{|c|}{$\mathrm{H}$} & \multicolumn{2}{|c|}{$\mathrm{N}$} & \multicolumn{2}{|c|}{ S } & \\
\hline & & & & & Cald & Found & Cald & Found & Cald & Found & Cald & Found & \\
\hline $5 a$ & $\mathrm{C}_{23} \mathrm{H}_{17} \mathrm{~N}_{3} \mathrm{OS}_{3}$ & 447 & 60 & $175-6$ & 61.17 & 61.05 & 3.8 & 3.8 & 9.31 & 9.3 & 21.47 & 21.3 & $\begin{array}{l}4.3\left(\mathrm{~s}, 1 \mathrm{H} \mathrm{C} \mathrm{C}_{2} \mathrm{H}, \mathrm{C}_{3} \mathrm{H}\right) \\
1.25\left(\mathrm{~s}, 2 \mathrm{H}, \mathrm{CH}_{2}\right)\end{array}$ \\
\hline $5 b$ & $\mathrm{C}_{24} \mathrm{H}_{19} \mathrm{~N}_{3} \mathrm{OS}_{3}$ & 475 & 50 & $180-1$ & 58.1 & 58.0 & 4.0 & 3.9 & 8.84 & 8.7 & 20.21 & 20.1 & $\begin{array}{l}4.6\left(1 \mathrm{H} \mathrm{d} . \mathrm{C}_{3} \mathrm{H}\right), 125 \mathrm{~s} \\
1 \mathrm{H}, \mathrm{C}_{2} \mathrm{H} 2.4\left(3 \mathrm{H} \mathrm{s} \mathrm{CH}_{3}\right)\end{array}$ \\
\hline $5 c$ & $\mathrm{C}_{23} \mathrm{H}_{17} \mathrm{~N}_{3} \mathrm{O}_{2} \mathrm{~S}_{3}$ & 463 & 55 & $190-1$ & 59.61 & 59.5 & 3.67 & 3.6 & 9.07 & 9.00 & 20.73 & 20.6 & $\begin{array}{l}4.6\left(1 \mathrm{H} \mathrm{d} . \mathrm{C}_{3} \mathrm{H}\right) \\
3.4(3 \mathrm{H} \mathrm{s} \mathrm{OH}\end{array}$ \\
\hline $5 \mathrm{~d}$ & $\mathrm{C}_{23} \mathrm{H}_{17} \mathrm{~N}_{3} \mathrm{O}_{2} \mathrm{~S}_{3}$ & 463 & 70 & $173-4$ & 59.61 & 59.5 & 3.67 & 3.6 & 9.07 & 9.00 & 20.73 & 20.6 & $\begin{array}{l}4.6\left(1 \mathrm{H} \mathrm{d} . \mathrm{C}_{3} \mathrm{H}\right) \\
3.4(3 \mathrm{H} \mathrm{s} \mathrm{OH}\end{array}$ \\
\hline $5 e$ & $\mathrm{C}_{24} \mathrm{H}_{19} \mathrm{~N}_{3} \mathrm{O}_{2} \mathrm{~S}_{3}$ & 477 & 65 & $198-9$ & 60.37 & 60.2 & 3.98 & 3.9 & 8.8 & 8.7 & 20.12 & 20.0 & $\begin{array}{l}4.6\left(1 \mathrm{H} \mathrm{d} . \mathrm{C}_{3} \mathrm{H}\right), 125 \mathrm{~s} \\
1 \mathrm{H}, \mathrm{C}_{2} \mathrm{H} 3.4\left(3 \mathrm{H} \mathrm{s} \mathrm{OCH}_{3}\right)\end{array}$ \\
\hline $5 f$ & $\mathrm{C}_{24} \mathrm{H}_{17} \mathrm{~N}_{3} \mathrm{O}_{3} \mathrm{~S}_{3}$ & 491 & 45 & $208-9$ & 58.65 & 58.5 & 3.46 & 3.4 & 8.55 & 8.5 & 19.55 & 19.4 & $\begin{array}{l}4.6\left(1 \mathrm{H} \mathrm{d} . \mathrm{C}_{3} \mathrm{H}\right) \\
3.4(3 \mathrm{H} \mathrm{s} \mathrm{OH}, 2, \mathrm{~s}, 2 \mathrm{H} \text { of } \\
\left.\mathrm{CH}_{2}\right)\end{array}$ \\
\hline $5 \mathrm{~g}$ & $\mathrm{C}_{24} \mathrm{H}_{19} \mathrm{~N}_{3} \mathrm{O}_{3} \mathrm{~S}_{3}$ & 493 & 50 & $185-6$ & 58.41 & 58.3 & 3.85 & 3.8 & 8.51 & 8.4 & 19.47 & 19.3 & $\begin{array}{l}4.6\left(1 \mathrm{H} \mathrm{d} . \mathrm{C}_{3} \mathrm{H}\right), 125 \mathrm{~s} \\
1 \mathrm{H}, \mathrm{C}_{2} \mathrm{H} 3.4\left(3 \mathrm{H} \mathrm{s} \mathrm{OCH}_{3}\right)\end{array}$ \\
\hline $5 \mathrm{~h}$ & $\mathrm{C}_{25} \mathrm{H}_{21} \mathrm{~N}_{3} \mathrm{O}_{3} \mathrm{~S}_{3}$ & 507 & 45 & $192-3$ & 59.17 & 59.1 & 4.14 & 4.1 & 8.28 & 8.2 & 18.93 & 18.8 & $\begin{array}{l}4.6\left(1 \mathrm{H} \mathrm{d} . \mathrm{C}_{3} \mathrm{H}\right), 125 \mathrm{~s}, \\
1 \mathrm{H}, \mathrm{C}_{2} \mathrm{H} 2.5\left(4 \mathrm{H} \mathrm{q}_{2}\right), \\
2.0\left(6 \mathrm{H} \mathrm{t} \mathrm{CH}_{3}\right)\end{array}$ \\
\hline
\end{tabular}

* Uncorrected $\quad * *$ All the NMR spectra containing multiplate between 7.8 to 8.2 which assigned aromatic ring $+\mathrm{C}_{4} \mathrm{H}$ and thiazole $\mathrm{CH}$ 
Table 3. Antifungal Activity of compounds 4a-h

\begin{tabular}{ccccc}
\hline \multirow{2}{*}{ Compound } & \multicolumn{4}{c}{ Zone of inhibition at $1000 \mathrm{ppm}(\%)$} \\
\cline { 2 - 5 } & $\begin{array}{c}\text { Penicillium } \\
\text { Expansum }\end{array}$ & $\begin{array}{c}\text { Botrydepladia } \\
\text { Thiobromine }\end{array}$ & Nigrospora Sp. & $\begin{array}{c}\text { Trichothesiun } \\
\text { Sp. }\end{array}$ \\
\hline 4a & 65 & 70 & 65 & 53 \\
4b & 77 & 85 & 75 & 74 \\
4c & 85 & 80 & 80 & 73 \\
4d & 81 & 81 & 79 & 83 \\
4e & 79 & 82 & 79 & 75 \\
4f & 78 & 80 & 72 & 71 \\
4g & 75 & 72 & 66 & 59 \\
4h & 74 & 70 & 61 & 66 \\
Penicillin & 85 & 65 & 75 & 75 \\
\hline
\end{tabular}

Table. 4 Antifungal Activity of compounds 5a-h

\begin{tabular}{ccccc}
\hline \multirow{2}{*}{ Compound } & \multicolumn{5}{c}{ Zone of inhibition at 1000 ppm $(\%)$} \\
\cline { 2 - 5 } & $\begin{array}{c}\text { Penicillium } \\
\text { Expansum }\end{array}$ & $\begin{array}{c}\text { Botrydepladia } \\
\text { Thiobromine }\end{array}$ & $\begin{array}{c}\text { Nigrospora } \\
\text { Sp. }\end{array}$ & $\begin{array}{c}\text { Trichothesiun } \\
\text { Sp. }\end{array}$ \\
\hline 5a & 64 & 71 & 65 & 75 \\
5b & 83 & 87 & 86 & 88 \\
5c & 78 & 85 & 75 & 80 \\
5d & 77 & 80 & 89 & 87 \\
5e & 74 & 80 & 65 & 74 \\
5f & 71 & 79 & 60 & 74 \\
5g & 72 & 72 & 62 & 72 \\
5h & 70 & 73 & 80 & 71 \\
Sulphanilamide & 85 & 75 & 70 & 85 \\
\hline
\end{tabular}

\section{Preparation of 2-Thiazolidinones $(5 a-h)$}

\section{General Procedure}

A mixture of Schiff base (3a-h) $(0.01 \mathrm{mmol})$ in THF $(30 \mathrm{ml})$ and mercapto acetic acid $(0.01 \mathrm{mmol})$ with a pinch of anhydrous $\mathrm{ZnCl}_{2}$ was then refluxed to at a residue, which was dissolved in 1,4-dioxane-ethanol mixture passed through a column of silica gel using benzene : chloroform $(8: 2, \mathrm{v} / \mathrm{v})$ mixture as an eluent. The eluent was concentrated and the product recrystallized 4-thiazolidinoines (5a-h) from ethanol: 1,4-dioxane (1:1) mixture $50-60 \%$ yield.The analytical data of all the compounds $4 \mathrm{a}-\mathrm{i}$ and $5 \mathrm{a}-\mathrm{i}$ are furnished in Tables 1 and 2.

\section{Results and Discussion}

2-amino-6-naphthalenylthiazolo [3,2-d] thiadiazole was prepared according to method reported for benzthiazole from aniline ${ }^{17}$. The elemental contents and IR-NMR spectral 
features shown in experimental section consistent with the predicted structure. The 2-amino6-naphthalenylthiazolo [3,2-d] thiadiazole (2) was dissolved in dioxane:ethanol (50:50) and was reacted with aromatic aldehyde in the presence of piperidine to yield Schiff bases (3ah). This Schiff bases (3a-h) were then characterized by the elemental analysis, IR spectral studies and NMR spectral studies. The IR spectra of Schiff bases show the prominent band at $1630 \mathrm{~cm}^{-1}$ for the azomethine group ${ }^{18-20}$.

These Schiff bases on cyclo-condensation reaction with chloro acetyl chloride afford 2azetidinone (4a-h) and with thio-glycolic acid afford 4-thiazolidinone (5a-h) respectively. The structures of both these compounds $(4 \mathrm{a}-\mathrm{h})$ and $(5 \mathrm{a}-\mathrm{h})$, respectively, have been confirmed by elemental analysis, IR spectral studies, and NMR spectral studies. These compounds shows the band at $1690 \mathrm{~cm}^{-1}$ for cyclic $>\mathrm{C}=\mathrm{O}$ group ${ }^{17}, 18$. All the compounds show the NMR signals for different kinds of protons at their respective positions. The data are shown in Tables 1 and 2 . The $\mathrm{C}, \mathrm{H}, \mathrm{N}, \mathrm{S}$ analysis of all the compounds of the series are presented in Tables 1 and 2. The values are consistent with their predicted structure (Scheme 1).

The antifungal activity of both the series (4a-h) and (5a-h), respectively, have been carried out against some strain of fungi. The results show that the prepared compounds are toxic against the bacteria. Compound $4 \mathrm{c}, 4 \mathrm{~d}, 4 \mathrm{~b}, 5 \mathrm{~b}, 5 \mathrm{~d}$ and $5 \mathrm{f}$ were found more active against the above fungi. The comparison of the antibacterial activity of these compounds with penicillin and sulphanilamide shows that these compounds have almost similar activity.

\section{References}

1. Coppola K, PTC Int.Appl. wo, 2001, 01108523.

2. Bian W, Jian Y N, Yang P, Xizng S H, Xuebzo S P and ZKexyeban, 2001,23 ,231, C.A. $134,237419$.

3. Patil S and Bhagaval G, J. Int. Char. Soc. 1994, 71, 205.

4. Manian A K, Khadse G G and Sengupta S R, Indian drugs, 1993, 30, 324.

5. Joshi M M, Bhagavat V S and Parvati J A, J. Ind. Chem. Soc. 1995, 70, 647.

6. Stepanor D E, Evanov E I and Evanov R Y, Russ. J.Char., 2000, 70 (5), 784.

7. Sharma R C and Kumar D, J. Ind. Chem. Soc., 2000, 77,492.

8. Joshi H D, Sawale A R, Ingle R D, and Mane R A, Ind. J. Chem., 2000, 39, 967.

9. Ingle V S, Sawale A R, Ingle R D, and Mane R A, Ind. J. Chem., 2001, 40, 124.

10. Kagathara P, Upadhyay T, Doshi R and Parekh H H, Indian J. Heterocycl. Chem., 2000, 10,9.

11. Matsui N, Jpn. Kokai Tokkyo JP, ,2000, 07, 652; Chem. Abstr., 2000, 132, 641094.

12. Desai K R, Asian J. Chem. Abstr, 2000, 132,279145.

13. Thaker K M, Ind. J. Chem., 2003, 42B, 1544.

14. Patel K H and Mehta A G, E- J.Chem., 2006, 11, 109.

15. Tripathy H and Mahaputra G N, J. Ind. Chem. Soc., 1975, 52,168.

16. (a) Barry A L, The antimicrobial susceptibility test: Principle and practices, $4^{\text {th }}$ ed. Philandelphia, 1976 (b) Bio. Abstr. 1977, 64, 25, 183.

17. Rangekar D W and Chaudhari M B, Dyes and Pigment, 1938, 10,173.

18. Kemp W, Organic Spectroscopy, $2^{\text {nd }}$ Edition. Basingtake, Macmillan, USA.

19. Furniss B, Hannaford A J, Smith P W G and Fatehell A R, Vage's Psnelial organic chemistry, Pearson Edition (Singapore) Pte.Ltd. Indian branch N.D 2004.

20. Panizzi J C, Davidovis G, Guglielmelti R, Mille G, Metzer J and Chateau J, Can. J. Chem., 1971, 49,956. 


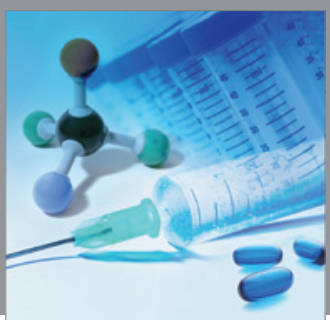

International Journal of

Medicinal Chemistry

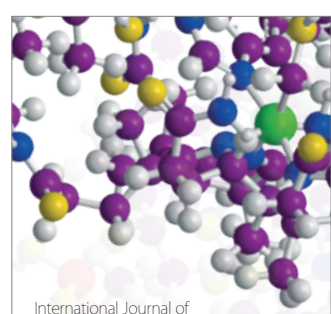

Carbohydrate Chemistry

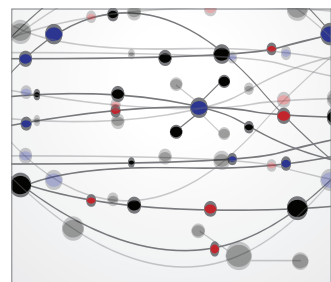

The Scientific World Journal
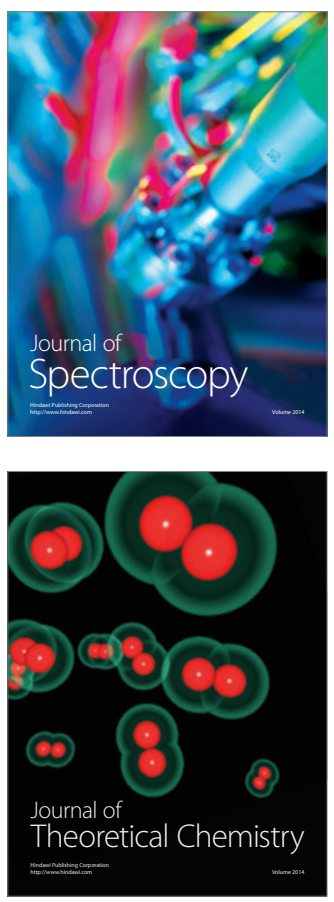
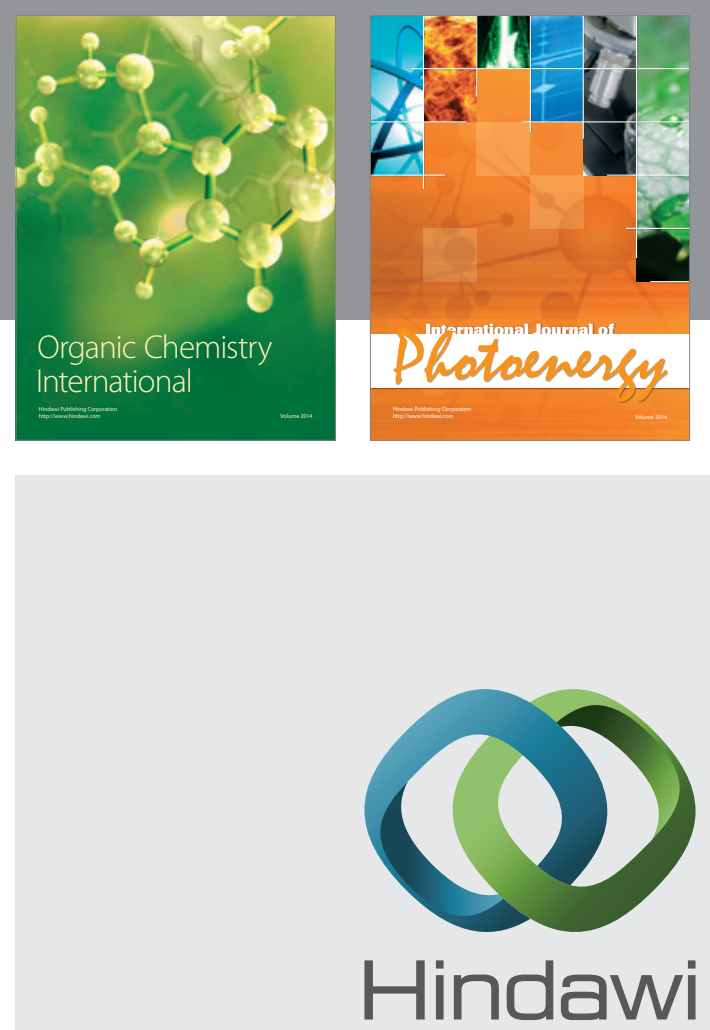

Submit your manuscripts at

http://www.hindawi.com
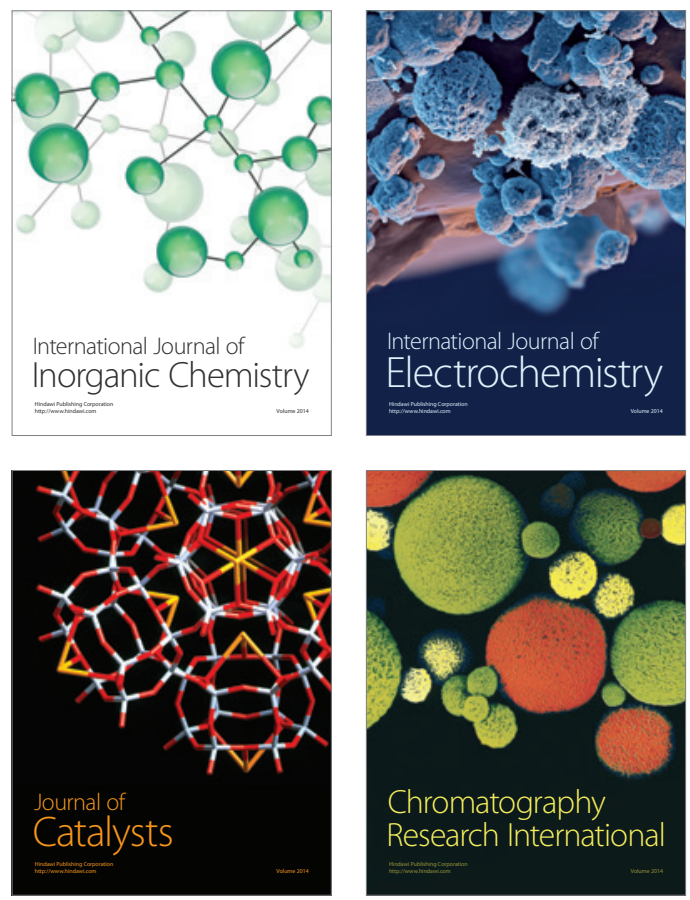
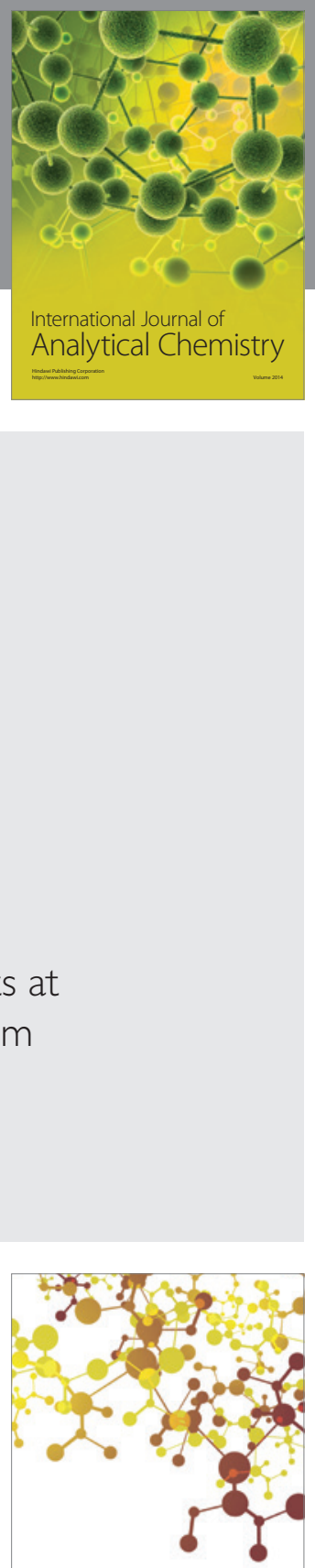

Journal of

Applied Chemistry
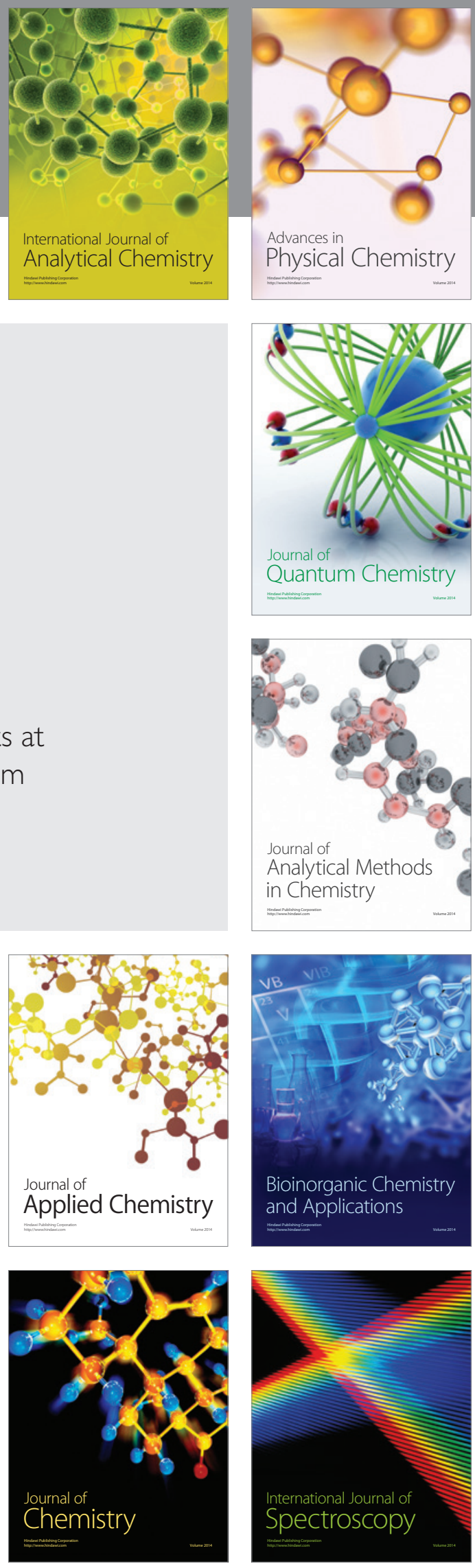